\title{
Effects of cigarette smoking on the growth of Streptococcus mutans biofilms: An in vitro study
}

Ye Han $\oplus^{*}$

Department of Preventive Dentistry, School of Dentistry, Institute of Oral Bioscience and BK 21 Plus Program, Jeonbuk National University, Jeonju, Republic of Korea

* hanye1747@gmail.com

\section{Abstract}

The increased incidence of dental caries by cigarette smoking (CS) has been widely reported in epidemiological studies, but the relationship between CS and cariogenic biofilm growth has been rarely studied. This study aims to investigate the effects of CS exposure on the growth and virulence of Streptococcus mutans biofilms (S. mutans). Briefly, S. mutans biofilms were formed on saliva-coated hydroxyapatite disks, which were exposed to CS 1,3 , and 6 times per day, respectively. In addition, S. mutans biofilms without CS exposure were considered as the control group. Acidogenicity, dry weight, colony-forming units (CFUs), water-soluble/insoluble extracellular polysaccharides (EPSs), and intracellular polysaccharides (IPSs) were analyzed and confocal laser scanning microscopy (CLSM) images of 74h-old S. mutans biofilms were obtained. The lowest accumulation of biofilms and EPSs were detected in the 6 times/day CS exposure group compared with those of the control group and other CS exposure groups in 74-h-old $S$. mutans biofilms. CLSM also revealed the lowest bacterial count (live and dead cells) and EPSs biovolume in the six times/day CS exposure group in 74-h-old $S$. mutans biofilms. CS exposure inhibited the growth of $S$. mutans biofilm in vitro study, the anti-cariogenic biofilm formation was enhanced with a dose (frequency)-dependent at which frequency has more influence in the present findings.

\section{Introduction}

Dental caries is a dental biofilm-related disease [1]. An important contributing factor in the development of dental caries is the change of microbial properties in dental biofilm. The increase of aciduric and acidogenic bacteria in the microorganism is of great significance in the pathogenesis of dental caries [1]. Through glycolysis, acidogenic bacteria reduce $\mathrm{pH}$ in the dental biofilm developed on the surface of teeth that are exposed to dietary sugar [1]. The low $\mathrm{pH}$ environment further accelerates the growth of acidity and acidogenic bacteria including mutans streptococci, other acidogenic streptococci, lactobacilli, and bifidobacteria [2]. Although the composition of cariogenic biofilm microflora is complex [3], S. mutans is considered one of the important etiological agents for the development of dental caries $[4,5]$. S. mutans can synthesize EPSs produce glucosyltransferases with sucrose as a substrate. EPSs contribute to bacterial adhesion on the tooth surface, colonization, and formation of plaque biofilm, which 
are important cariogenic factors of S. mutans. Besides, acid production through carbohydrates and acid tolerance in low $\mathrm{pH}$ is also the major cariogenic factors of $S$. mutans that result in the loss of local hard tissue and the initiation of the cariogenic process [6].

CS is an important factor affecting the progress of dental caries. Numerous epidemiological studies around the world have reported a close relationship between smoking and the occurrence of dental caries [7-11]. An animal study reported that CS exposure expands the cariesaffected area in the maxillary molars of the rat [12]. This is further confirmed by evidence from epidemiological studies associating CS with high caries prevalence [13].

To the best of the author's knowledge, the relationship between CS and the growth of cariogenic biofilm (S. mutans biofilms) has been rarely analyzed, which is of great significance to study the influence of CS on the growth of $S$. mutans biofilms and dynamic bacterial equilibrium for further understanding the relationship between smoking and high caries prevalence. This study aimed to investigate the influence of CS on the growth, virulence (EPSs and acidogenicity), and viability of cariogenic biofilms in vitro by using the $S$. mutans biofilm model.

\section{Materials and methods}

\section{S. mutans biofilms formation and CS experimental scheme}

S. mutans UA159 (ATCC 700610; serotype c) biofilms were formed on saliva-coated hydroxyapatite discs $\left(2.93 \mathrm{~cm}^{2}\right.$; Clarkson Chromatography Products, Inc., South Williamsport, PA, USA) placed in a vertical position in 24-well plates. Briefly, an adult male (non-smoker) was selected for oral saliva collection. Hydroxyapatite discs were incubated in filter-sterilized $\left(0.22-\mu \mathrm{m}\right.$ low protein-binding filter) saliva $(3 \mathrm{ml} / \mathrm{disc})$ for $1 \mathrm{~h}$ at $37^{\circ} \mathrm{C}$. For biofilms formation, the saliva-coated hydroxyapatite discs were transferred to a 24 -well plate containing brain heart infusion (BHI; D-ifco, Detroit, MI, USA) broth with $1 \%$ (w/v) sucrose and S. mutans (5$7 \times 10^{6}$ colony-forming unit $\left.(\mathrm{CFU}) / \mathrm{ml}\right)(3 \mathrm{ml} / \mathrm{disc})$. The biofilms were grown at $37^{\circ} \mathrm{C}$ with $5 \%$ $\mathrm{CO}_{2}$ until $74 \mathrm{~h}$ (Fig 1). After $21 \mathrm{~h}$ (initial biofilms growth), S. mutans biofilms were divided

\section{S. mutans biofilms start} $5-7 \times 10^{6} \mathrm{CFUs} / \mathrm{ml}$

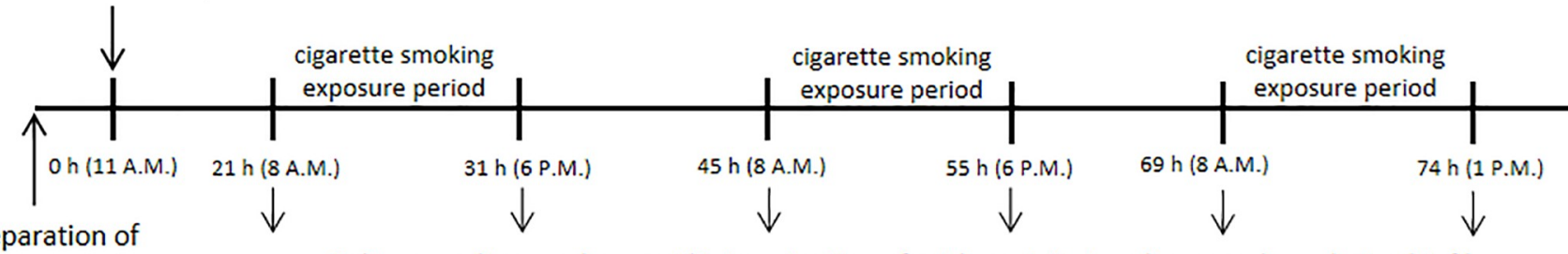

saliva-coated hydroxyapatite discs
Culture medium replacment/Determination of acidogenicity in culture medium during biofilm formation ( $\mathrm{pH}$ values of old culture medium)

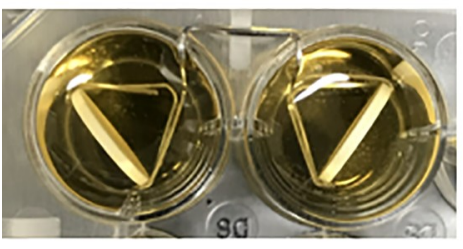

S. mutans biofilms on saliva-coated hydroxyapatite discs placed in a vertical position in a 24-well plate
Effects of cigarette smoking on $S$. mutans biofilms during biofilms formation. - Dry weight CFUs count Water-insoluble/soluble EPSs, IPSs Acidogenicity Bacterial and EPS biovolume and thickness by confocal laser scanning microscopy

Fig 1. S. mutans biofilms formation and experimental scheme. 
into 4 groups. Experiment group 1 (control group) did not receive CS exposure and are exposed to the air six times per day (at 8, 10, 12 a.m., 2, 4, 6 p.m.). Experiment group 2 was exposed one time per day (at 10 a.m.) with CS and five times per day (at 8, 12 a.m., 2, 4, 6 p.m.) with air, a total of three times CS exposure in 74-h-old biofilms. Experiment group 3 was exposed three times per day (at 10 a.m., 2, 6 p.m.) with CS and three times per day (at 8, 12 a. m., 4 p.m.) with air, a total of seven times with CS exposure in 74-h-old biofilms. Experiment group 4 was exposed six times per day (at 8, 10, 12 a.m., 2, 4, 6 p.m.) with CS, a total of fifteen times in 74-h-old biofilms. Each exposure time was 5 minutes (simulate the real smoking time of smokers). The culture medium was changed twice daily (8 a.m. and 6 p.m.) (Oral sugar levels rise after 8 a.m. for breakfast and 6 p.m. for dinner). The hydroxyapatite disks were washed with distilled water three times a day ( 8 a.m., 1 p.m., 6 p.m.) (Simulate cleaning mouth after breakfast, lunch, and dinner) for the control group and all CS exposure groups. This study is approved by the ethics committee/institutional review board of the Department of Preventive Dentistry, School of Dentistry, Institute of Oral Bioscience, Jeonbuk National University. All experimental protocols were approved by the Department of Preventive Dentistry, School of Dentistry, Institute of Oral Bioscience, Jeonbuk National University. The author confirms that all methods were carried out by relevant guidelines and regulations and informed consent had been obtained from the subject.

The microenvironment of plaque is easily affected by various bacteria and external factors. To minimize the influence of external factors and improve the internal validity of the results, and in vitro method was considered. Considering that $S$. mutans is a facultative anaerobe, a glass container was designed to allow slight air to enter while CS was inhaled to avoid the effect of complete hypoxia on the growth of $S$. mutans biofilm. At the time of exposure, the salivacoated hydroxyapatite discs were taken out from the culture medium, placed in a sterile glass container, and a cigarette was taken using a vacuum machine to simulate cigarette gas in the mouth during smoking, each exposure time was 5 minutes. After exposure, saliva-coated hydroxyapatite discs were returned to the culture medium. This device simulates CS flow has been verified in a previous study (Fig 2) [14].

In this experiment, a popular cigarette in Korean supermarkets was selected. Marlboro (tar: $8.0 \mathrm{mg}$; nicotine: $0.7 \mathrm{mg}$ ), has the highest tar and nicotine content per cigarette of all cigarette brands. We used vacuum machines to provide smoking force.

\section{Microbiological and biochemical biofilm analyses}

The dry weight and CFUs in the homogenized suspension were analyzed. Briefly, the 74-h-old biofilms on the saliva-coated hydroxyapatite disc were transferred into $2 \mathrm{ml}$ of $0.89 \% \mathrm{NaCl}$ and sonicated in an ultrasonic bath for $10 \mathrm{~min}$ to disperse the biofilms. The dispersed solution was re-sonicated at $7 \mathrm{~W}$ for $30 \mathrm{~s}$ after adding $3 \mathrm{ml}$ of $0.89 \% \mathrm{NaCl}$ (VCX 130PB; Sonics and Materials, Inc., Newtown, CT, USA). For the determination of CFUs count, an aliquot (0.1 ml) of the homogenized solution $(5 \mathrm{ml})$ was serially diluted, plated onto brain heart infusion (BHI; Difco, Detroit, MI, USA) agar plates, and then incubated under aerobic conditions at $37^{\circ} \mathrm{C}$ to determine the CFUs count $[15,16]$.

For the determination of the dry weight and amount of water-insoluble/soluble EPSs and IPSs, the remaining solution $(4.9 \mathrm{ml})$ was centrifuged $(3000 \times \mathrm{g})$ for $20 \mathrm{~min}$ at $4^{\circ} \mathrm{C}$. The biofilm pellet was resuspended and washed twice in the same volume of water. Mix the water-washed the biofilms pellet with $95 \%$ alcohol and put it in a refrigerator at $-20^{\circ} \mathrm{C}$ for at least $18 \mathrm{~h}$ to precipitate the water-soluble extracellular polysaccharides (EPSs). Then calculate the content of water-soluble EPSs in the biofilms. The washed biofilms pellet was evenly divided into two portions, lyophilized, and weighed to determine the dry weight. One part used $1 \mathrm{~N}$ sodium 


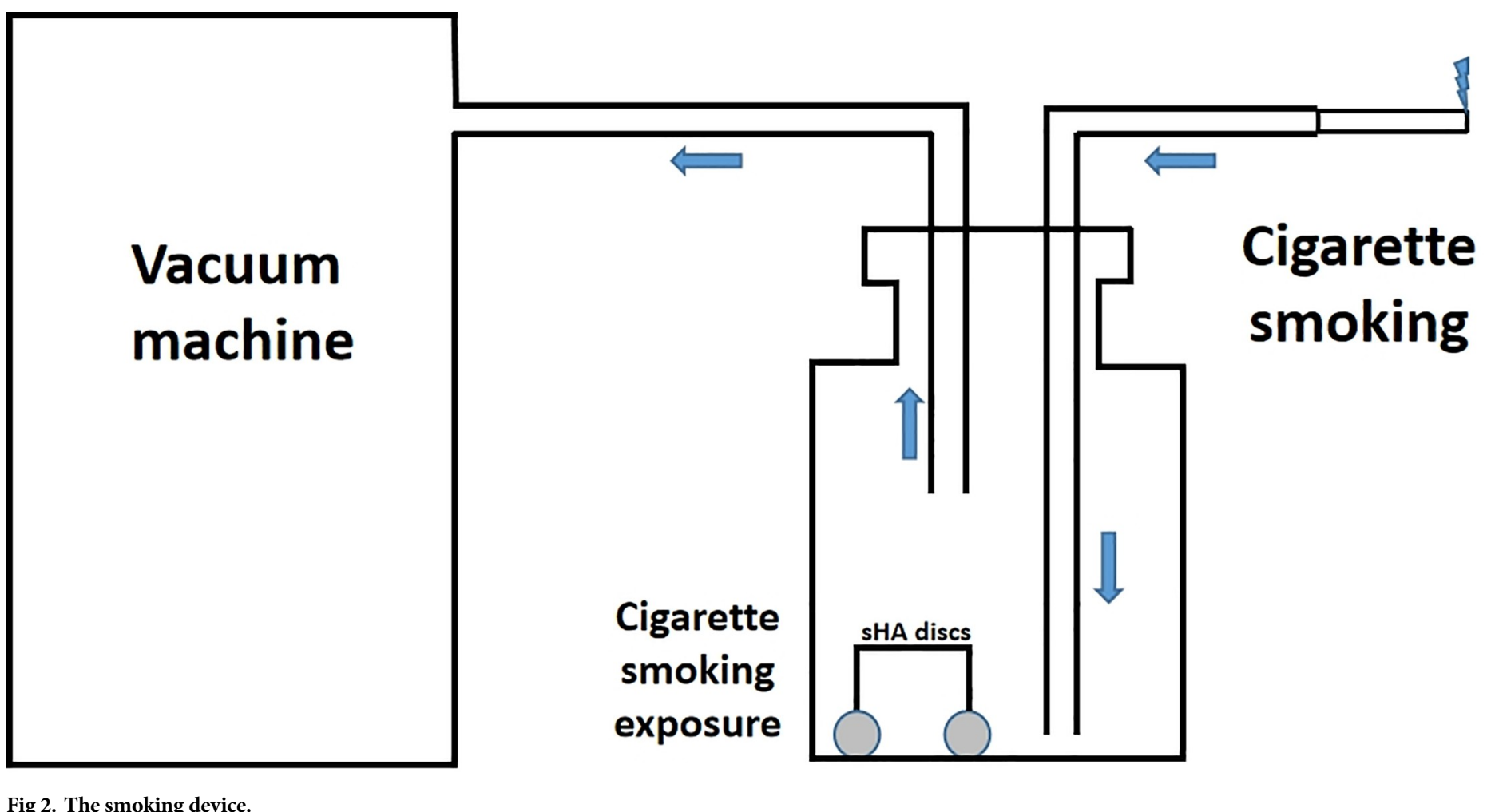

https://doi.org/10.1371/journal.pone.0259895.g002

hydroxide to extract water-insoluble EPSs from the dried precipitate. The other part was used to calculate the content of intracellular polysaccharides (IPSs), as detailed elsewhere [17].

The final $\mathrm{pH}$ values of the old culture media were also determined during the experimental period using a glass electrode (Beckman Coulter Inc., Brea, CA, USA) to investigate the change in acidogenicity of $S$. mutans biofilms by the CS exposure.

\section{CLSM analysis}

Live and dead bacterial cells staining. CLSM analysis was performed to confirm the results of microbiological and biochemical studies. To investigate the difference in bacterial cells, the 74-h-old biofilms were stained at room temperature in the dark for 30 min using the Film Tracer LIVE/DEAD Biofilm viability kit L10316 (Invitrogen, Molecular Probes Inc., Eugene, OR, USA). The final concentrations of SYTO $® 9$ and propidium iodide (PI) were 6.0 and $30 \mu \mathrm{M}$, respectively. This viability kit was based on plasma membrane integrity to determine live and dead cells. In this study, we regarded the cells with intact membranes (green) as live cells, whereas cells with damaged membranes (red) were regarded as dead cells. The excitation/emission wavelengths were $480 / 500 \mathrm{~nm}$ for $\mathrm{SYTO} \AA 9$ and $490 / 635 \mathrm{~nm}$ for PI for collecting the fluorescence. The stained live and dead bacterial cells were observed with an LSM 510 META microscope (Carl Zeiss, Jena, Germany) equipped with argon-ion and helium-neon lasers. All confocal fluorescence images were taken with an EC Plan-Neofuar 10x/0.30 M27 objective lens. A stack of slices in $6.4 \mu \mathrm{m}$ step sizes was captured from the top to the bottom of the biofilms. The biovolume and thickness of live and dead cells were quantified from the entire stack using COMSTAT image-processing software. The biovolume is defined as the volume of the biomass $\left(\mu \mathrm{m}^{3}\right)$ divided by the substratum (hydroxyapatite surface) area $\left(\mu \mathrm{m}^{2}\right)$. The three-dimensional architecture of the biofilms was visualized using ZEN 2.3 (blue edition) 
(Carl Zeiss Microscopy GmbH, Jena, Germany). The original confocal data was uploaded to ZEN 2.3 software and the intensity of green and red fluorescence in the full thickness of biofilms layers were captured automatically. The software reconstructed the 2-dimensional intensity of fluorescence in all the layers to a 3-dimensional volume stack [18].

EPS staining. The EPSs of 74-h-old biofilms were also investigated by simultaneous in situ labeling as described elsewhere [19]. Briefly, Alexa Fluor $\AA$ 647-labeled dextran conjugate ( $1 \mu \mathrm{M}$, 10,000 MW; absorbance/fluorescence emission maxima 647/668 nm; Molecular Probes Inc., Eugene, OR, USA) was added to the culture medium during the formation of $S$. mutans biofilms (at 0, 21, 31, 45, 55, $69 \mathrm{~h}$ ) to label the newly formed EPSs. As described above, the stained EPSs were observed with an LSM 510 META microscope (Carl Zeiss, Jena, Germany) (objective: EC Plan Neofuar 10x/0.30 M27) equipped with argon-ion and helium-neon lasers and visualized using ZEN 2.3. A stack of slices in $7.8 \mu \mathrm{m}$ step sizes was captured from the top to the bottom of the biofilms. Four independent experiments were performed, and five image stacks per experiment were collected. The EPSs biovolume and thickness were quantified from the confocal stacks using COMSTAT.

\section{Biofilm density}

The density of the 74-h-old S. mutans biofilms were calculated using the dry weight, which was derived from the biochemical study above, and the total biovolume of the biofilms (live cells + dead cells + EPSs), which was derived from the CLSM study above. The biofilm density $\left(\mu \mathrm{g} / \mu \mathrm{m}^{3}\right)$ is defined as the dry weight $\left(\mu \mathrm{g} / \mu \mathrm{m}^{2}\right)$ divided by the total biovolume of the biofilms $\left(\mu \mathrm{m}^{3} / \mu \mathrm{m}^{2}\right)$ [20].

\section{Statistical analysis}

All experiments (except CLSM and SEM) were performed in duplicate, and at least six different experiments were conducted. The data are presented as mean \pm standard deviation. Intergroup differences were estimated using a one-way analysis of variance, followed by a post hoc multiple comparisons (Tukey) test to compare multiple means (IBM SPSS Statistics for Windows, version 17) (IBM Corp., Armonk, n.y., USA). Values were considered statistically significant when the $p$-value was $<0.05$.

\section{Result}

\section{The difference in S. mutans biofilms formation}

74-h-old S. mutans biofilms formation was inhibited by CS exposure. In the experimental results of microbial and biochemical studies, all CS exposure groups reduced the dry weight accumulation, water-insoluble/soluble EPSs, and IPSs formation of the 74-h-old S. mutans biofilms in a frequency-dependent manner, and the lowest values in the six times/day CS exposure group compared with the control group and other CS exposure groups (Fig 3A-3E, $p<0.05$ ). There was no significant difference in bacterial activity (CFUs) counts among all CS exposure groups (Fig 3B; $p>0.05$ ).

\section{The $\mathrm{pH}$ value in the culture medium}

Fig 4 shows the $\mathrm{pH}$ change in the culture medium by different times of CS exposure during 74-h-old S. mutans biofilms formation. Acidogenicity during S. mutans biofilms formation was affected by CS exposure. Although the acidogenicity of $S$. mutans biofilms before the CS exposure was not different from each other $(p>0.05)(0 \sim 21 \mathrm{~h}$ of biofilm formation). But the $\mathrm{pH}$ in the culture medium started to change after exposure with CS, the acidogenicity was inhibited at all of the CS exposure groups $(p<0.05)(21 \sim 74 \mathrm{~h})$. The inhibition of acidogenicity of biofilms was enhanced with the increase of CS exposure frequency, and the highest 

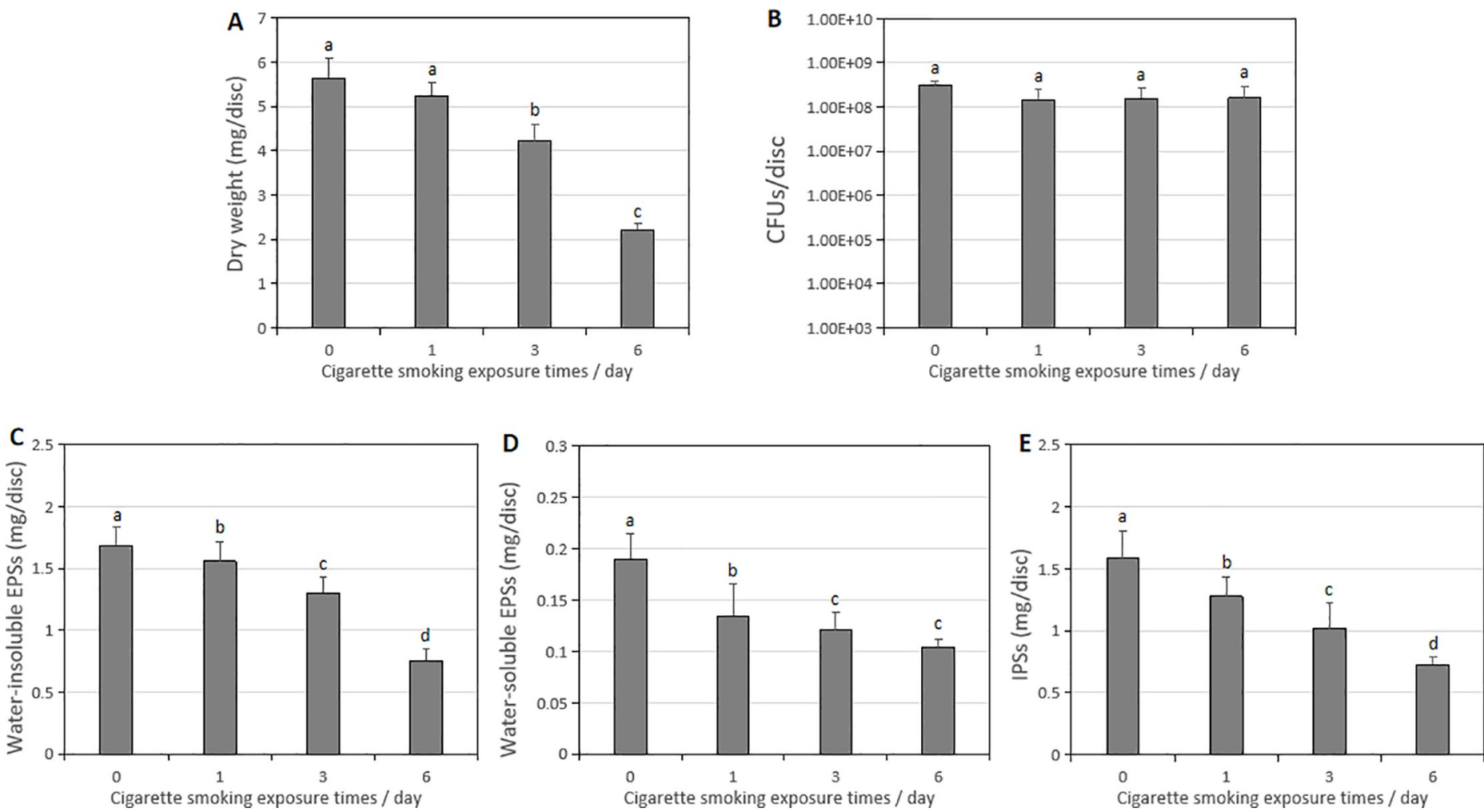

Fig 3. Change in the microbiological and biochemical composition of the 74-h-old S. mutans UA159 biofilms exposure with CS. (A) Dry weight. (B) CFUs. (C) Water-insoluble EPSs. (D) Water-soluble EPSs. (E) IPSs. Data represent mean \pm standard deviation. Fig 3A and 3C and 3D and 3E, $p<0.05$. Fig 3B, $p>0.05^{*} p<0.05$ : significantly different from each other. $p>0.05$ : values followed by the same superscript are not significantly different from each other.

https://doi.org/10.1371/journal.pone.0259895.g003

inhibition ability was shown in the six times/day CS exposure group $(p<0.05)$. Furthermore, in all of the CS exposure periods and non-CS exposure periods $(21 \sim 74 \mathrm{~h})$, the $\mathrm{pH}$ values of the culture medium of $S$. mutans biofilms in the six times/day CS exposure group were significantly different from the control group $(p<0.05)$.

\section{Bacterial biovolume and thickness}

To further evaluate the effect of CS on biofilm components and structure, the CLSM analysis was performed. The bacterial biovolume and thickness of live or dead cells of the 74-h-old $S$. mutans biofilms showed different results under different times of CS exposure (Fig 5). The lowest values of bacterial biovolume and thickness of live or dead cells and total biovolume of the were detected in the six times/day CS exposure group compared with the other CS exposure groups and control group in the 74-h-old S. mutans biofilms (Fig 5A and 5B and 5D; $p<0.05)$. In the percent of total biovolume, the proportion of live cell biovolume in each exposure group was between $60 \%$ and $70 \%$ (Fig 5E). The three-dimensional image of bacterial microcolonies of 74-h-old S. mutans biofilms showed that with the increased frequency of CS exposure, the morphology was changed into a lower number of the live/dead cells microcolonies with a smaller size and a loose arrangement (Fig 5C).

\section{EPS biovolume and thickness}

The biovolume and thickness of EPSs in the 74-h-old S. mutans biofilms are influenced by the different times of CS exposure. The mean biovolume and thickness of EPSs of the 74-h-old $S$. 


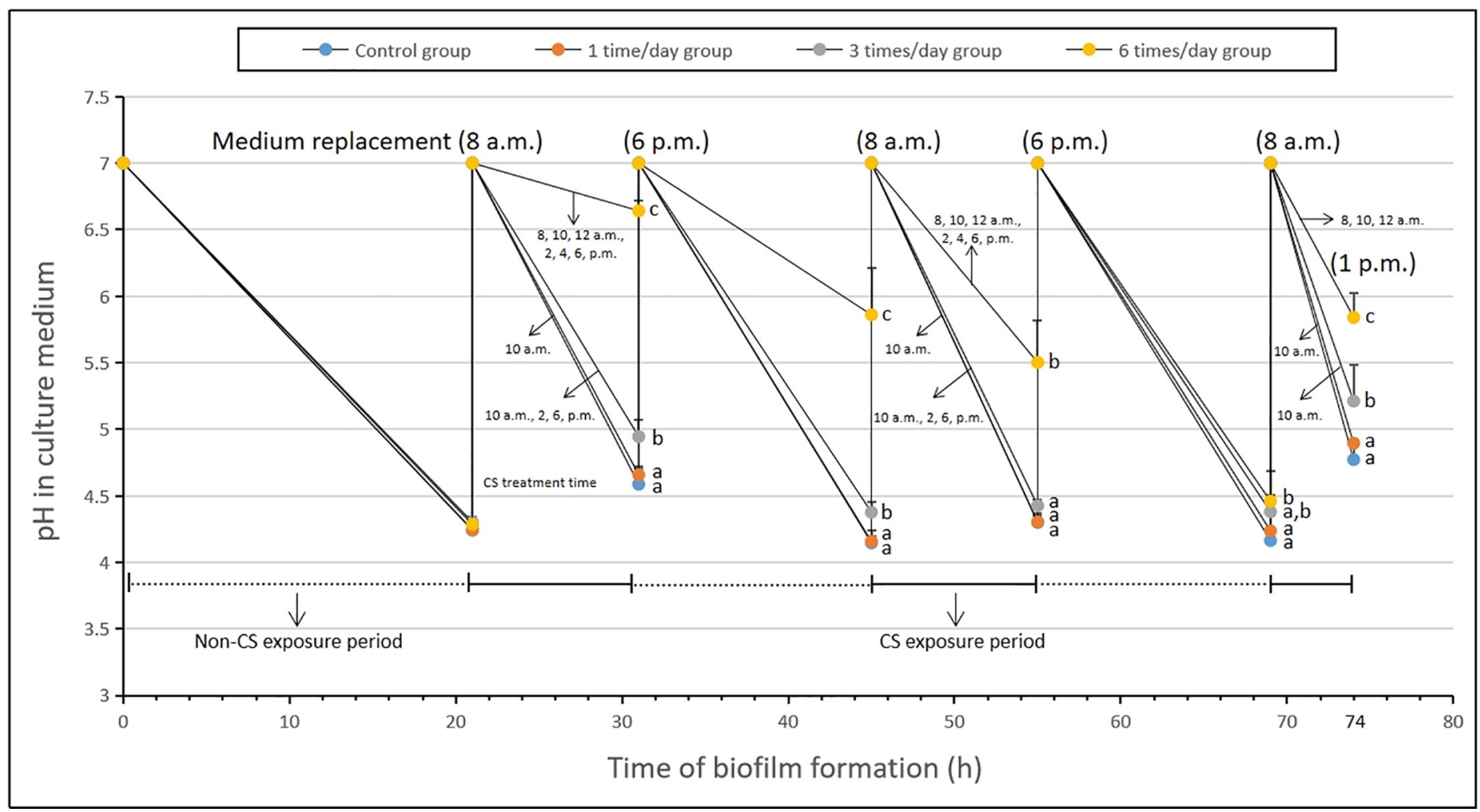

Fig 4. Change in the $\mathrm{pH}$ values of old culture medium exposure with CS during 74-h-old $S$. mutans UA159 biofilms formation. Data represent mean \pm standard deviation. Values followed by the same superscript are not significantly different from each other $(p>0.05)$.

https://doi.org/10.1371/journal.pone.0259895.g004

mutans biofilms in the six times/day CS exposure group showed the lowest values compared with the other CS exposure groups and control group (Fig 6A and 6B; $p<0.05$ ). With the increase of CS exposure frequency, the anti-EPSs formation increased in a frequency-dependent manner. EPSs and bacterial microcolony images of 74-h-old S. mutans biofilms showed minimal concentrations of homogeneous structures of EPSs covering and surrounding bacterial microcolonies in the six times/day CS exposure group (Fig 6C).

\section{The difference in biofilm density}

The density of $S$. mutans biofilms were calculated to investigate the difference in biofilm compactness according to different times of CS exposure. As shown in Fig 7, there was no significant difference in biofilm density in all the CS exposure groups and the control group $(p>0.05)$.

Fig 8 showed the surface morphology of each group of hydroxyapatite disks after 74-h-old incubation. The surface of the hydroxyapatite disk in the six times/day CS exposure group showed the largest area of the yellow film (a mixture of chemical substances produced by cigarette combustion) covering compared to the other CS exposure groups and the control group. And the accumulation of biofilm on the surface of the yellow film covering the area was less than that on the surface of white hydroxyapatite.

\section{Discussion}

This study was to investigate the differences in growth, virulence (EPSs and acidogenicity), and viability of cariogenic biofilms according to the different times of CS exposure (simulate 

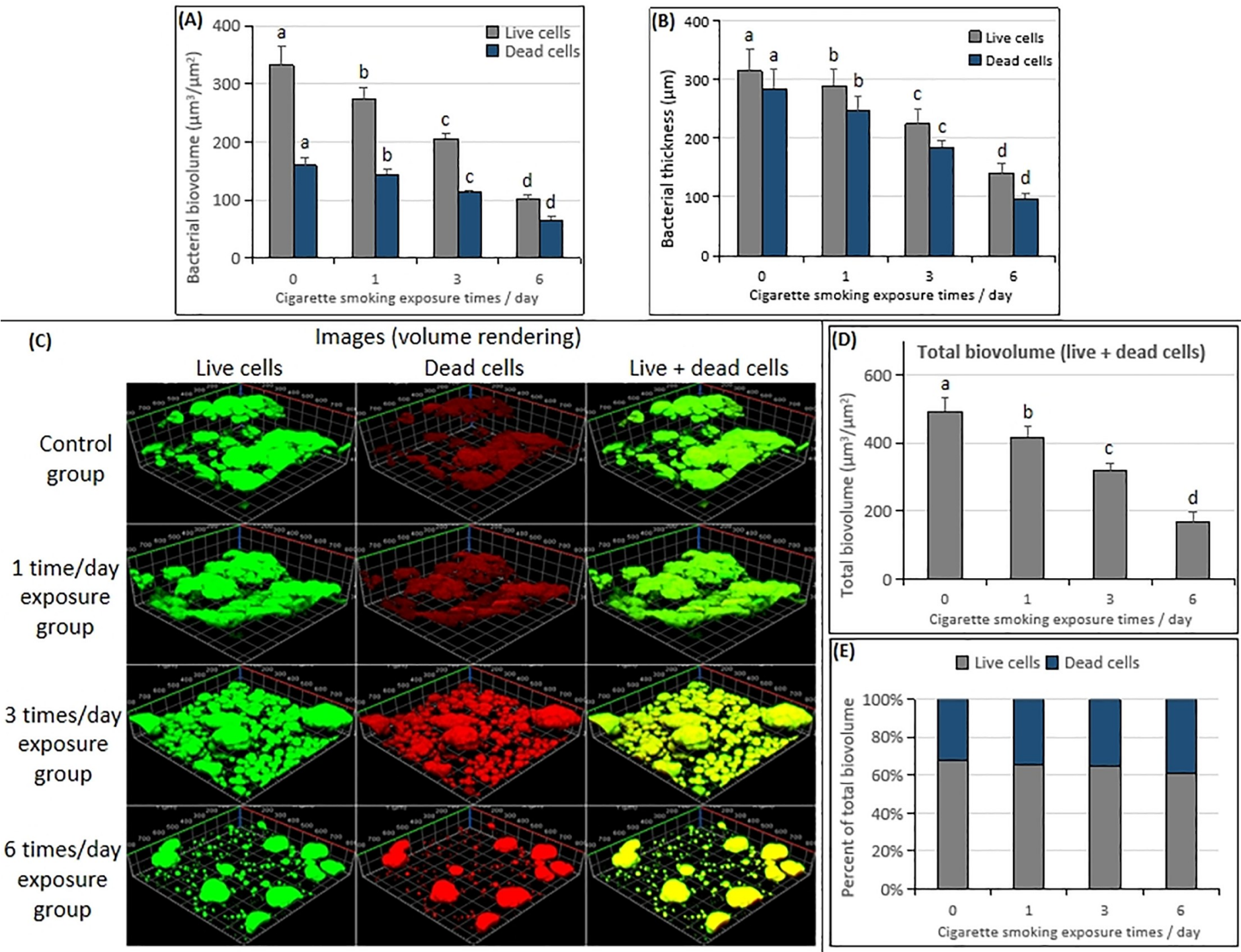

Fig 5. Change in CLSM of bacterial cells in the 74-h-old S. mutans UA159 biofilms exposure with CS. (A) Bacterial biovolume. (B) Bacterial thickness. (C) Representative confocal images. (D) Total biovolume (live + dead cells). (E) Percent of the total biovolume. Significantly different from each other $(p<0.05)$.

https://doi.org/10.1371/journal.pone.0259895.g005

different smoking times each day) using an S. mutans biofilm model in vitro. S. mutans biofilm model is unable to precisely mimic the complex microbial community in dental biofilms, the mono-species biofilm is advantageous in examining the mechanisms of $S$. mutans in biofilms, in biofilms with sufficient performance to validate the data reproducibility and reduce variance [17, 21, 22].

As shown in Fig 3A, all CS exposure groups reduce the dry weight accumulation of 74-hold S. mutans biofilm. The six times/day CS exposure group showed the lowest dry weight accumulation compared with the control group and other CS exposure groups (Fig 3A; $p<0.05)$. CS inhibited the dry weight accumulation of $S$. mutans biofilm in a frequencydependent manner. There was no significant difference in the bacterial activity between all CS exposure groups and the control group (Fig 3B; $p>0.05$ ). CLSM revealed that the live/dead cells bacterial biovolume, bacterial thickness, and total biovolume in 74-h-old S. mutans biofilms were frequency-dependently reduced by CS exposure (Fig 5A and 5B and 5D; $p<0.05$ ). 


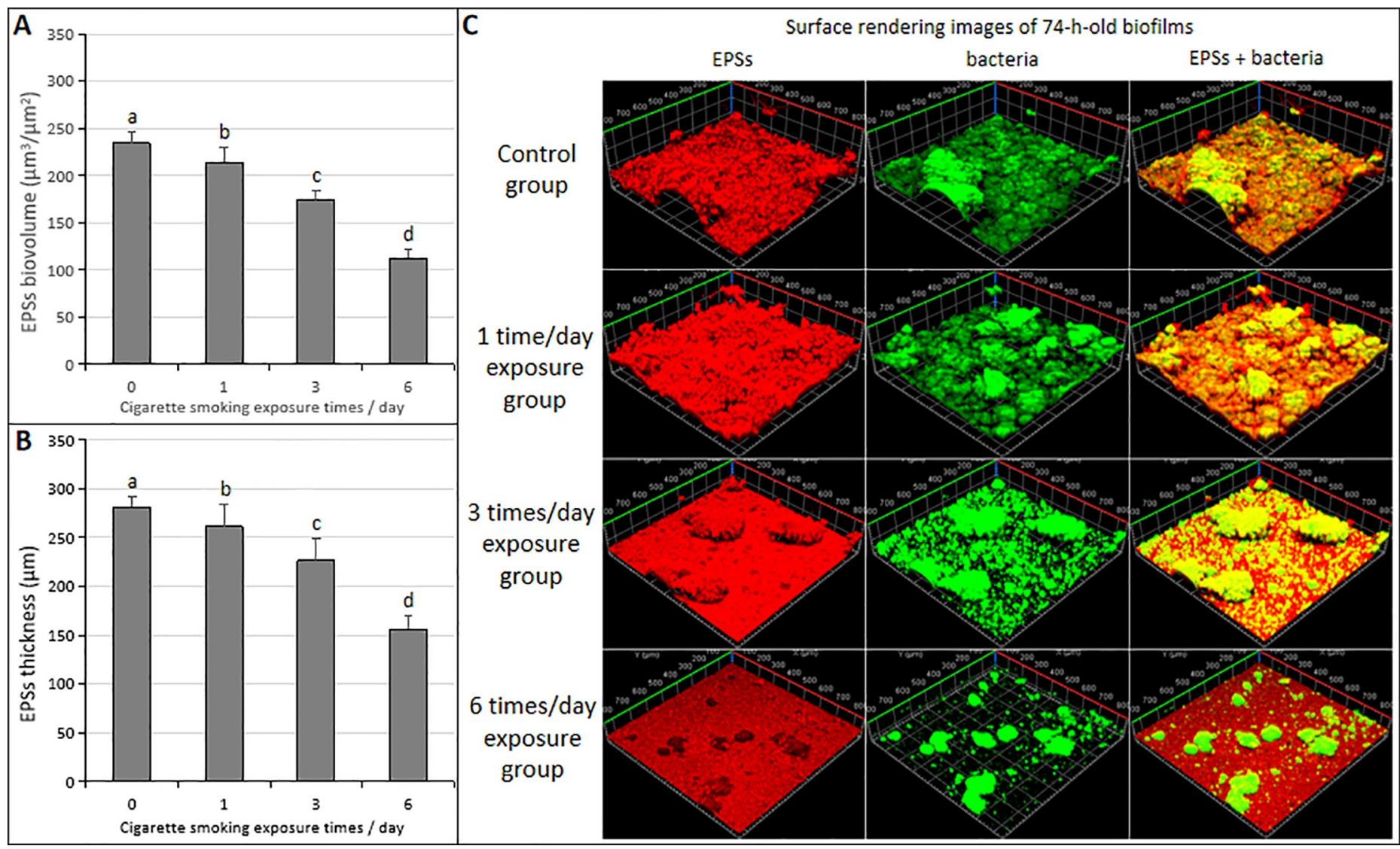

Fig 6. Change in CLSM of EPSs in the 74-h-old S. mutans UA159 biofilms exposure with CS. (A) EPSs biovolume. (B) EPSs thickness. (C) Representative confocal images. Significantly different from each other $(p<0.05)$.

https://doi.org/10.1371/journal.pone.0259895.g006

The lowest values were shown in the six times/day CS exposure group. With the increase of CS exposure times, the number of live/dead cell microcolonies decreased and the arrangement of microcolonies was loose. However, the morphology of microcolonies did not change significantly, microcolonies in each treatment group that were similar to those in the control group could be observed (Fig 5C). In addition, according to the results of the bacterial activity (Fig $3 \mathrm{~B}$ ), the live/dead cell microcolonies (Fig 5C), and the proportion of live cell biovolume (Fig 5E), the CS exposure had no bactericidal or killing effect on the growth of S. mutans biofilms.

Polysaccharides account for up to $40 \%$ of the dry weight of dental biofilms is, which are mostly synthesized by microbial glucosyltransferases [23]. Therefore, the reduction of biofilm biomass is directly related to the reduction of polysaccharides in the whole biofilm matrix. The complex structural integrity of a biofilm is mainly determined by the density of the formation, volume, structural integrity, and stability of which is also associated with EPSs [6, 24]. In this study, the effects of CS on the synthesis of water-soluble/insoluble EPSs and IPSs during the formation of S. mutans biofilms were investigated. All the CS exposure groups in the 74-h-old S. mutans biofilms decreased the synthesis of water-soluble/insoluble EPSs and IPSs, the lowest values were detected in the six times/day CS exposure group compared to the control group and other CS exposure groups (Fig 3C-3E; $p<0.05$ ). CS exposure inhibited the synthesis of polysaccharides of 74-h-old $S$. mutans biofilm in a frequency-dependent manner, which was consistent with the CLSM findings of the biovolume and thickness of EPSs (Fig 6; $p<0.05$ ). Therefore, CS had an obvious damage effect on the EPSs formation of biofilms, and the 


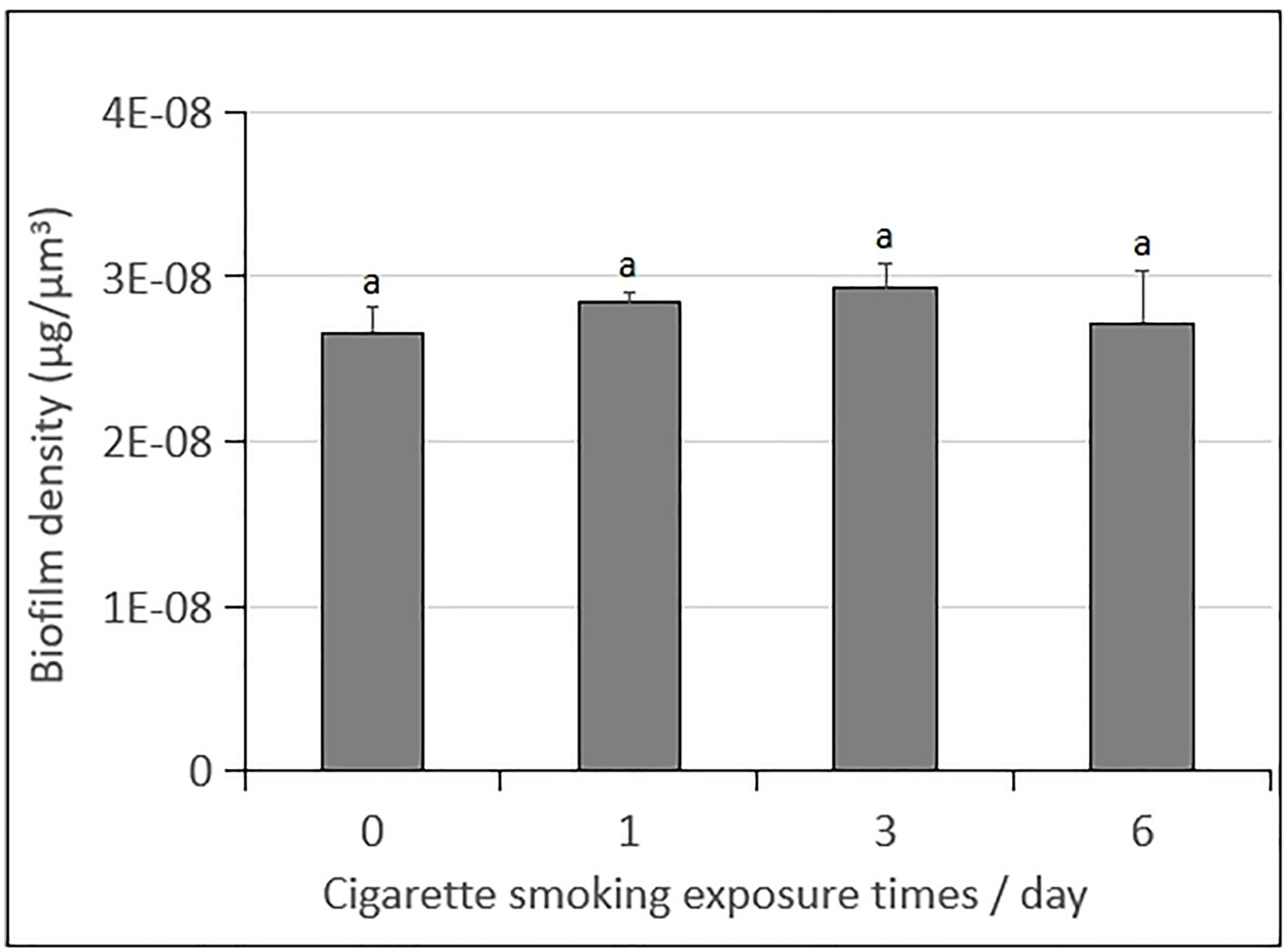

Fig 7. Change in the biofilm density of 74-h-old S. mutans UA159 biofilms exposure with CS. Values followed by the same superscripts are not significantly different from each other $(p>0.05)$.

https://doi.org/10.1371/journal.pone.0259895.g007

decreased biovolume and thickness of EPSs may affect the growth height of bacterial cells (Fig 6).

Acid production by carbohydrate and acid tolerance in a low $\mathrm{pH}$ environment is one of the main toxicity characteristics of $S$. mutans [6], which are closely associated with enamel demineralization and the formation of dental caries [25-27]. In this study, acid production of $S$. mutans biofilms was found to be inhibited by all CS exposure groups through analyzing $\mathrm{pH}$ values in the old culture medium, and the highest inhibitory rate was detected in the six times/ day CS exposure group (Fig $4 ; p>0.05$ ).

The above results indicated that the CS exposure inhibited the growth of $S$. mutans biofilms in a frequency-dependent manner in vitro, which was well supported by the measurements of biofilm dry weight (Fig 3A), EPSs (Figs 3C and 3D and 6), IPSs (Fig 3E), acidogenic (Fig 4), bacterial count (live or dead cells) (Fig 5A) and total biovolume (Fig 5D). The six times/day CS exposure group showed the highest inhibition compared to the control group, and other CS exposure groups. No significant differences in the bacterial activity (CFUs counts; Fig 3B) and 


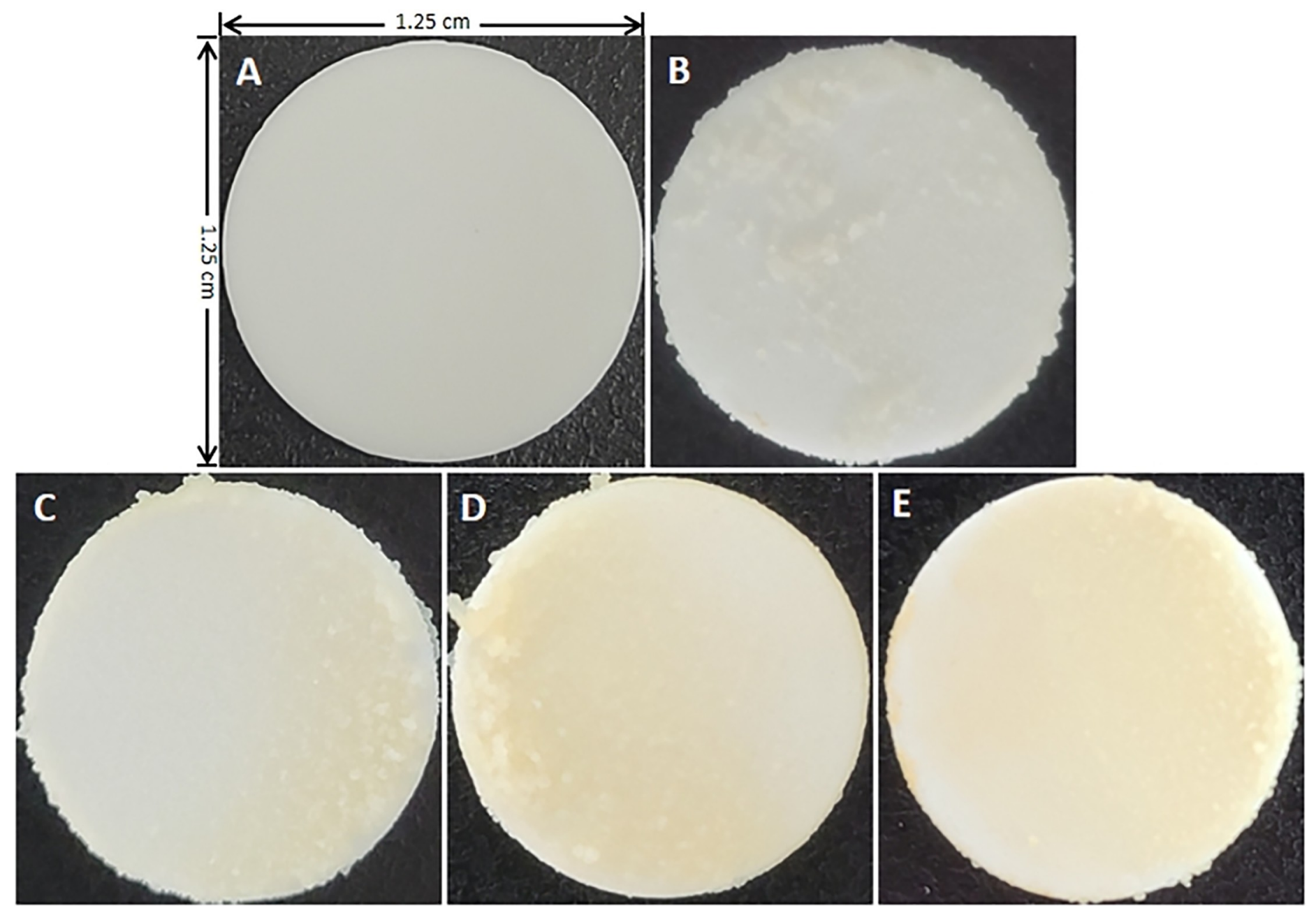

Fig 8. Surface morphology of hydroxyapatite disks before the experiment and each group after 74-h-old incubation. (A) Before the experiment. (B) Control group. (C) 1 time/day CS exposure group. (D) 3 times/day CS exposure group. (E) 6 times/day CS exposure group.

https://doi.org/10.1371/journal.pone.0259895.g008

biofilm density (Fig 7) were detected between all CS exposure groups and the control group. Meanwhile, CLSM revealed a similar ratio of live cells and dead cells in the total biovolume between all CS exposure groups and the control group (Fig 5E). These results suggested that CS did not inhibit the growth of $S$. mutans biofilms by sterilizing or killing cells.

The author further analyzed the mechanism of CS exposure in inhibiting the growth of $S$. mutans biofilm in vitro. It is considered that CS exposure altered the colonization environment on the surface of the hydroxyapatite disc where S. mutans biofilms were attached. The hydroxyapatite disks in all CS treatment groups at $74 \mathrm{~h}$ incubation showed that the surface of the hydroxyapatite disks was covered with a yellow film by the mixture of chemical substances produced by cigarette combustion attached to the surface of the hydroxyapatite disks (Fig 8). Cigarette combustion produces more than five thousand chemicals that contain various harmful substances [28-30]. The area of the yellow film on the surface of the hydroxyapatite disk was significantly larger in the six times/day exposure group than that of other CS exposure groups and the control group. Compared with the normal white hydroxyapatite disk in the control group, the accumulation of biofilms on the yellow film surface in the six times/day exposure group was less than that on the normal hydroxyapatite disk surface, and the formation of $S$. 
mutans biofilms could not even be seen on some areas of the yellow film surface (Fig 8). This may be since the mixture of chemical substances produced by cigarette combustion attached to the surface of the hydroxyapatite disc, altered the environment of the initial hydroxyapatite disc that blocked the colonization of S. mutans biofilms on the surface of the chemical mixture. This study showed that with the increased frequency of CS exposure, the novel mixture of chemical substances produced by cigarette combustion continuously covered the surface of $S$. mutans biofilms, inhibited the growth and the new colonization of S. mutans biofilm on the surface of the chemical mixture. In the hydroxyapatite disks of the CS exposure groups, with the increased frequency of CS exposures, the available area for the colonization and growth of S. mutans biofilms became smaller and smaller. As a result, the lowest biofilm dry weight, EPSs, IPSs, acidogenic bacterial count (live or dead cells), and total biovolume were detected in the six times/day CS exposure group. CLSM revealed that the thickness of bacteria (live or dead cells) was negatively correlated with the frequency of CS exposures, The lowest bacterial thickness detected in the six times/day CS exposure group also confirmed that the coverage of mixture chemical substances produced by cigarette combustion continuously covered on the surface of the S. mutans biofilms inhibited the increase in bacterial thickness (Fig 5B). Mixture chemical substances produced by cigarette combustion that were attached to the surface of the hydroxyapatite disk also resulted in the color change of the disk. In practice, the mixture of chemical substances of CS attached to the surface of the teeth caused the color change [31].

The results of this study support CS exposure in inhibiting $S$. mutans biofilms growth was inconsistent with previous ones that smoking is closely related to the high incidence of dental caries [7-13], which could be explained by this study is in vitro and lacked the influence of the oral immune system on the growth process of cariogenic biofilm. One study demonstrated that salivary secretory $\operatorname{IgA}(\mathrm{s}-\operatorname{IgA}), \mathrm{pH}$, and flow rates were of great significance in oral mucosal immunity, and salivary S-IgA antibodies generated by the mucosal immune system were an important role in the immune response against dental caries. Low concentrations of salivary sIgA were correlated with a higher prevalence of dental caries in smokers [32]. It has been shown that CS impairs saliva function, which has an important role in promoting caries. In addition, both plasma and saliva activities of lactic dehydrogenase (LDH), aspartate aminotransferase (AST), and amylase are significantly reduced by external addition of aldehydes or CS exposure [33]. A decreased buffering effect of the saliva of smokers, and higher numbers of Lactobacilli and S. mutans increase the susceptibility to dental caries [34], which may explain the relationship between CS and the high incidence of dental caries.

In general, the results in vitro study supported the fact that CS exposure inhibited the growth of $S$. mutans biofilms because the mixture of chemical substances produced by cigarette combustion changed the colonizing environment on the surface of the hydroxyapatite disc, rather than sterilizing or killing cells. In this study, the frequency of CS exposure was the main variable, and there was no interference from other oral factors. Although only one cariogenic bacterium was included in this study, these findings provide a novel insight into clarifying the biological effects of CS on cariogenic biofilm growth.

\section{Conclusion}

CS exposure inhibited the growth of $S$. mutans biofilm in vitro study, the anti-cariogenic biofilm formation was enhanced with a dose (frequency)-dependent at which frequency has more influence in the present findings.

\section{Author Contributions}

Writing - original draft: Ye Han. 
Writing - review \& editing: Ye Han.

\section{References}

1. Marsh PD. Are dental diseases examples of ecological catastrophes? Microbiology. 2003; 149: 279294. https://doi.org/10.1099/mic.0.26082-0 PMID: 12624191

2. Marsh PD. Controlling the oral biofilm with antimicrobials. J Dent. 2010; 38: 11-15. https://doi.org/10. 1016/S0300-5712(10)70005-1 PMID: 20621238

3. Takahashi N, Nyvad B. Caries ecology revisited: microbial dynamics and the caries process. Caries Res. 2008; 42: 409-418. https://doi.org/10.1159/000159604 PMID: 18832827.

4. Hojo K, Nagaoka S, Ohshima T, Maeda N. Bacterial interactions in dental biofilm development. J Dent Res. 2009; 88: 982-990. https://doi.org/10.1177/0022034509346811 PMID: 19828884

5. Lemos JA, Quivey RG, Koo H, Abranches J. Streptococcus mutans: a new Gram-positive paradigm? Microbiology. 2013; 159: 436-445. https://doi.org/10.1099/mic.0.066134-0 PMID: 23393147

6. Bowen $\mathrm{WH}, \mathrm{Koo} \mathrm{H}$. Biology of Streptococcus mutans-derived glucosyltransferases: role in extracellular matrix formation of cariogenic biofilms. Caries Res. 2011; 45: 69-86. https://doi.org/10.1159/ 000324598 PMID: 21346355

7. Campus G, Cagetti MG, Senna A, Blasi G, Mascolo A, Demarchi P, et al. Does Smoking Increase Risk for Caries? A Cross-Sectional Study in an Italian Military Academy. Caries Res. 2011; 45: 40-46. https://doi.org/10.1159/000322852 PMID: 21228593

8. Nobre MdA, Maló P. Prevalence of periodontitis, dental caries, and peri-implant pathology and their relation with systemic status and smoking habits: Results of an opencohort study with 22009 patients in a private rehabilitation center. Journal of Dentistry. 2017; 67: 36-42. https://doi.org/10.1016/j.jdent. 2017.07.013 PMID: 28750777

9. Bernabé E, Delgado-Angulo EK, Vehkalahti MM, Aromaa A, Suominen AL. Daily smoking and 4-year caries increment in Finnish adults. Community Dent Oral Epidemiol. 2014; 42: 428-434. https://doi.org/ 10.1111/cdoe.12101 PMID: 24476541

10. Bernabé E, MacRitchie H, Longbottom C, Pitts NB, Sabbah W. Birth Weight Breastfeeding, Maternal Smoking and Caries Trajectories. Journal of Dental Research. 2017; 96: 171-178. https://doi.org/10. 1177/0022034516678181 PMID: 27834298

11. Aguilar-Zinser V, Irigoyen ME, Rivera G, Maupomé G, Sánchez-Pérez L, Velázquez C. Cigarette Smoking and Dental Caries among Professional Truck Drivers in Mexico. Caries Res. 2008; 42: 255-262. https://doi.org/10.1159/000135670 PMID: 18523384

12. Fujinami $Y$, Nakano K, Ueda O, Ara T, Hattori $T$, Kawakami T, et al. Dental Caries Area of Rat Molar Expanded by Cigarette Smoke Exposure. Caries Res. 2011; 45: 561-567. https://doi.org/10.1159/ 000331926 PMID: 22067411

13. Shenkin JD, Broffitt B, Levy SM, Warren JJ. The association between environmental tobacco smoke and primary tooth caries. Journal of Public Health Dentistry. 2004; 64: 184-186. https://doi.org/10 1111/j.1752-7325.2004.tb02750.x PMID: 15341143

14. Cuadr GA, Smith MT, Nelson JM, Loh EK, Palazzolo DL. A Comparison of Flavorless Electronic Cigarette-Generated Aerosol and Conventional Cigarette Smoke on the Survival and Growth of Common Oral Commensal Streptococci. Int J Environ Res Public Health. 2019; 16: 1669. https://doi.org/10 3390/ijerph16101669

15. Koo H, Schobel B, Scott-Anne K, Watson G, Bowen WH, Cury JA, et al. Apigenin and tt-farnesol with fluoride effects on S. mutans biofilms and dental caries. J Dent Res. 2005; 84: 1016-1020. https://doi. org/10.1177/154405910508401109 PMID: 16246933

16. Pandit S, Cai JN, Song KY, Jeon JG. Identification of anti-biofilm components in Withania somnifera and their effect on virulence of Streptococcus mutans biofilms. J Appl Microbiol. 2015; 119: 571-581. https://doi.org/10.1111/jam.12851 PMID: 25976122

17. Lemos JA, Abranches J, Koo H, Marquis RE, Burne RA. Protocols to study the physiology of oral biofilms. Methods Mol Biol. 2010; 666: 87-102. https://doi.org/10.1007/978-1-60761-820-1_7 PMID: 20717780

18. Zhang T, Wang ZJ, Hancock REW, Fuente-Núñez CDL, Haapasalo M. Treatment of Oral Bioflms by a D-Enantiomeric Peptide. PLoS One. 2016; 23: e0166997. https://doi.org/10.1371/journal.pone. 0166997

19. Hwang G, Liu Y, Kim DY, Sun V, Aviles-Reyes A, Kajfasz JK, et al. Simultaneous spatiotemporal mapping of in situ pH and bacterial activity within an intact 3D microcolony structure. Sci. Rep. 2016; 6: 32841. https://doi.org/10.1038/srep32841 PMID: 27604325 
20. Dang MH, Jung JE, Choi HM, Jeon JG. Difference in virulence and composition of a cariogenic biofilm according to substratum direction. Sci. Rep. 2018; 8: 6244. https://doi.org/10.1038/s41598-018-246262 PMID: 29674703

21. Klein MI, DeBaz L, Agidi S, Lee H, Xie G, Lin AHM. Dynamics of Streptococcus mutans transcriptome in response to starch and sucrose during biofilm development. PLoS One. 2010; 5: e13478. https://doi. org/10.1371/journal.pone.0013478 PMID: 20976057

22. Pandit S, Kim JE, Jung KH, Chang KW, Jeon JG. Effect of sodium fluoride on the virulence factors and composition of Streptococcus mutans biofilms. Archives of oral biology. 2011; 56: 643-649. https://doi. org/10.1016/j.archoralbio.2010.12.012 PMID: 21241981

23. Koo H, Xiao J, Klein MI, Jeon JG. Exopolysaccharides produced by Streptococcus mutans glucosyltransferases modulate the establishment of microcolonies within multispecies biofilms. J Bacteriol. 2010; 192: 3024-3032. https://doi.org/10.1128/JB.01649-09 PMID: 20233920

24. Koo H, Jeon JG. Naturally occurring molecules as alternative therapeutic agents against cariogenic biofilms. Adv Dent Res. 2009; 21: 63-68. https://doi.org/10.1177/0895937409335629 PMID: 19717411

25. Kwon YR, Son KJ, Pandit S, Kim JE, Chang KW, Jeon JG. Bioactivity-guided separation of anti-acidogenic substances against Streptococcus mutans UA 159 from Polygonum cuspidatum. Oral Dis. 2010; 16: 204-209. https://doi.org/10.1111/j.1601-0825.2009.01636.x PMID: 20374506

26. Zhou L, Ding Y, Chen W, Zhang P, Chen Y, Lv X. The in vitro study of ursolic acid and oleanolic acid inhibiting cariogenic microorganisms as well as biofilm. Oral Dis. 2013; 19: 494-500. https://doi.org/10. 1111/odi.12031 PMID: 23114261

27. Gregoire S, Singh AP, Vorsa N, Koo H. Influence of cranberry phenolics on glucan synthesis by glucosyltransferases and Streptococcus mutans acidogenicity. J Appl Microbiol. 2007; 103: 1960-8. https:// doi.org/10.1111/j.1365-2672.2007.03441.x PMID: 17953606

28. Borgerding M, Klus H. Analysis of complex mixtures-cigarette smoke. Exp Toxicol Pathol. 2005; 57: 43-73. https://doi.org/10.1016/j.etp.2005.05.010 PMID: 16092717

29. Thielen A, Klus H, Muller L. Tobacco smoke: unraveling a controversial subject. Exp Toxicol Pathol. 2008; 60: 141-156. https://doi.org/10.1016/j.etp.2008.01.014 PMID: 18485684

30. Talhout R, Schulz T, Florek E, Benthem JV, Wester P, Opperhuizen A. Hazardous Compounds in Tobacco Smoke. Int J Environ Res Public Health. 2011; 8: 613-628. https://doi.org/10.3390/ ijerph8020613 PMID: 21556207

31. Jesper R. Tobacco and Oral Diseases. Med Princ Pract. 2003; 12: 22-32. https://doi.org/10.1159/ 000069845 PMID: 12707498

32. Benderli $\mathrm{Y}$, Erdilek $\mathrm{D}$, Koray $\mathrm{F}$, Telci $\mathrm{A}$, Turan $\mathrm{N}$. The relation between salivary IgA and caries in renal transplant patients. Oral surg. Oral Med. Oral Pathol. Oral Radiol. Endod. 2000; 89: 588-593. https:// doi.org/10.1067/moe.2000.105144 PMID: 10807716

33. Zappacosta B, Persichilli S, Mordente A, Minucci A, Lazzaro D, Meucci E, et al. Inhibition of salivary enzymes by cigarette smoke and the protective role of glutathione. Human Experimental Toxicology. 2002; 21: 7-11. https://doi.org/10.1191/0960327102ht202oa PMID: 12046726

34. Cogulu D, Sabah E, Kutukculer N, Ozkinay F. Evaluation of the relationship between caries indices and salivary secretory IgA, salivary $\mathrm{pH}$, buffering capacity and flow rate in children with Down's syndrome. Archives of Oral Biology. 2006; 51: 23-28. https://doi.org/10.1016/j.archoralbio.2005.06.001 PMID: 16039983 